Moroccan J. of Pure and Appl. Anal. (MJPAA)

Volume 5(1), 2019, Pages 31-36

ISSN: Online 2351-8227 - Print 2605-6364

DOI 10.2478/mjpaa-2019-0003

\title{
Fixed point theorem satisfying cyclical conditions in $b$-Menger spaces
}

\author{
AbderRAhim Mbarki ${ }^{1}$ AND RACHID OUbrahim ${ }^{2, a}$
}

AвstRAct. In this work, we prove a fixed point theorem for mapping with cyclical conditions using comparison function in $b$-Menger spaces. We support our results by an example.

2010 Mathematics Subject Classification. 54E70, 54H25, 47S50.

Key words and phrases. $b$-Menger space; fixed point; comparison function.

\section{Introduction}

The notion of statistical metric spaces, as a generalization of metric spaces, with non-deterministic distance, was defined by Menger [6] in 1942. An extension of Banach's contraction [1] in probabilistic metric spaces was showed by Sehgal and Bharucha-Reid [8] in 1972 and their fixed point theorem is further generalized by many authors, for example see [2,5].

Recently, Mbarki et al. [4] introduced the probabilistic $b$-metris spaces ( $b$-Menger spaces) as a generalization of probabilistic metric spaces (Menger spaces) and they studied topological structures and properties and showed the fixed point property for nonlinear contractions in these spaces.

In 2003, Kirk et al. [3] gave the cyclic contraction mappings as follows:

Let $A$ and $B$ be nonempty subsets of a metric space $(X, d)$. A mapping $T: A \cup B \rightarrow A \cup B$ is said a cyclic contraction mapping if we have

(1) $T(A) \subseteq B$ and $T(B) \subseteq A$.

(2) $d(T x, T y) \leq k d(x, y), \forall x \in A, \forall y \in B$, where $k \in[0,1)$.

And they proved that $T$ has a unique fixed point in $A \cap B$.

In this paper, we prove the existence and uniqueness of the fixed point for mapping with cyclical conditions using comparison function in a $b$-Menger spaces and we give an example which support the main result.

Received May 15, 2019 - Accepted July 21, 2019.

(C) The Author(s) 2017. This article is published with open access by Sidi Mohamed Ben Abdallah University.

${ }^{1}$ ANO Laboratory, National School of Applied Sciences, Mohammed First University, P.O. Box 669, Oujda, Morocco.

2 ANO Laboratory, Faculty of Sciences, Mohammed First University, 60000 Oujda, Morocco.

a e-mail: rchd.oubrahim@gmail.com . 


\section{Preliminaries}

Throughout this work, we adopt the usual terminology, notation and conventions of the theory of probabilistic $b$-metric spaces, as in [4]

Definition 2.1. A distance distribution function (briefly, a d.d.f.) is a nondecreasing function $F$ defined on $\mathbb{R}^{+} \cup\{\infty\}$ that satisfies $F(0)=0$ and $F(\infty)=1$, and is left continuous on $(0, \infty)$. The set of all d.d.f's will be noted by $\Delta^{+}$; and the set of all $F$ in $\Delta^{+}$for which $\lim _{t \rightarrow \infty} F(t)=1$ by $D^{+}$.

A simple example of distribution function is Heavyside function in $D^{+}$

$$
H(t)=\left\{\begin{array}{lll}
0 & \text { if } \quad t \leq 0 \\
1 & \text { if } t>0
\end{array}\right.
$$

A triangular norm (briefly, a $t$-norm) is a binary operation $T$ on $[0,1]$, which satisfies the following conditions:

(1) $T(x, y)=T(y, x)$, for all $x, y \in[0,1]$.

(2) $T(x, y) \leq T(z, w)$, whenever $x \leq z, y \leq w$, for each $x, y, z, w \in[0,1]$.

(3) $T(x, 1)=x$, for all $x \in[0,1]$.

(4) $T(T(x, y), z)=T(x, T(y, z))$, for all $x, y, z \in[0,1]$.

Example 2.1. The following $t$-norms are continuous:

(1) The t-norm minimum, $T_{M}(x, y)=\operatorname{Min}(x, y)$.

(2) The t-norm product, $T_{p}(x, y)=x y$.

(3) The t-norm $T_{L}, T_{L}(x, y)=\operatorname{Max}(x+y-1,0)$.

Definition 2.2. [4] $A b$-Menger space is a quadruple $(M, F, T, s)$ where $M$ is a nonempty set, $F$ is a function from $M \times M$ into $\Delta^{+}, T$ is a continuous t-norm, $s \geq 1$ is a real number, and the following conditions are satisfied: for all $p, r ; q \in M$ and $x, y>0$,

(1) $F_{p p}=H \quad$ (H is the Haeryside function),

(2) $F_{p r}=H \Rightarrow p=r$,

(3) $F_{p r}=F_{r p}$,

(4) $F_{p r}(s(x+y)) \geq T\left(F_{p q}(x), F_{q r}(y)\right)$.

It should be noted that a Menger space is a $b$-Menger with $s=1$.

Example 2.2. [4] Let $M=\{1,2,3,4\}$. Define $F: M \times M \rightarrow \Delta^{+}$as follow:

$$
F_{p q}(t)=\left\{\begin{array}{l}
H(t) \text { if } p=q, \\
H(t-3) \text { if }[p=2 \text { and } q=3] \text { or }[p=3 \text { and } q=2], \\
H(t-1) \text { otherwise. }
\end{array}\right.
$$

It is easy to check that $\left(M, F, T_{M}, 2\right)$ is a $b$-Menger space, and since:

$$
F_{32}\left(\frac{5}{2}\right)=0<1=H\left(\frac{1}{4}\right)=\operatorname{Min}\left(F_{31}\left(\frac{5}{4}\right), F_{12}\left(\frac{5}{4}\right)\right)
$$

So $\left(M, F, T_{M}\right)$ is not a standard Menger space.

Example 2.3. [4] Let $M=[0, \infty)$. Define $F: M \times M \rightarrow \Delta^{+}$as follow:

$$
F_{p q}(t)=H\left(t-|p-q|^{2}\right)
$$

It easy to check that $\left(M, F, T_{M}, 2\right)$ is a b-Menger space, but $\left(M, F, T_{M}\right)$ is not a standard Menger space since we have:

$$
F_{32}\left(\frac{2}{3}\right)=0<1=H\left(\frac{1}{12}\right)=\operatorname{Min}\left(F_{3 \frac{5}{2}}\left(\frac{1}{3}\right), F_{\frac{5}{2} 2}\left(\frac{1}{3}\right)\right)
$$


Definition 2.3. Let $\left\{x_{n}\right\}$ be a sequence in a b-Menger space $(M, F, T, s)$ and $x \in M$.

(1) A sequence $\left\{x_{n}\right\}$ is convergent to $x$, if

$$
\lim _{n \rightarrow \infty} F_{x_{n} x}(t)=1 \text { for all } t>0 .
$$

(2) A sequence $\left\{x_{n}\right\}$ is a G-Cauchy sequence if

$$
\lim _{n \rightarrow \infty} F_{x_{n+r} x_{n}}(t)=1 \text { for any } r>0 \text { and } t>0 .
$$

(3) The b-Menger space $(M, F, T, s)$ is said to be G-complete if every G-Cauchy sequence is convergent.

Definition 2.4. Let $(M, F)$ be a probabilistic semimetric space (i.e., ( $i)$, (ii) and (iii) of Definition 2.2 are satisfied). For $p$ in $M$ and $t>0$, the strong $t$-neighborhood of $p$ is the set

$$
N_{p}(t)=\left\{q \in M: F_{p q}(t)>1-t\right\} .
$$

The strong neighborhood system at $p$ is the collection

$$
\wp_{p}=\left\{N_{p}(t): t>0\right\},
$$

and the strong neighborhood system for $M$ is the union

$$
\wp=\bigcup_{p \in M} \wp_{p} .
$$

Remark 2.1. Let $(M, F)$ be a probabilistic semimetric space.

(1) $\forall p \in M, N_{p}\left(t_{1}\right) \subset N_{p}\left(t_{2}\right)$ whenever $t_{1} \leq t_{2}$

(2) Since $\wp_{p}$ and the collection of neighborhoods $\left\{N_{p}\left(\frac{1}{n}\right): n=1,2, \ldots\right\}$ are equivalent at every point in $M, \wp$ is first-countable.

Mbarki et al.[4] proved that if $(M, F, T, s)$ is a $b$-Menger space with $T$ is continuous, then the family $\Im$ consisting of $\varnothing$ and all unions of elements of this strong neighborhood system for $M$ determines a topology for $M$. Moreover, we have the following assertions

(1) $(M, F, T, s)$ endowed with the topology $\Im$ is a Hausdorff space provided that $T$ is continuous.

(2) The function $F$ is in general not continuous.

We confirm the last assertion by the following example

Example 2.4. [4] Let $M=\mathbb{N} \cup\{\infty\}, 0<a \leq 1$. Define $F^{a}: M \times M \rightarrow \Delta^{+}$as follow:

$$
F_{p q}^{a}(t)=\left\{\begin{array}{l}
H(t) \text { if } p=q, \\
H(t-7) \text { if } p \text { and } q \text { are odd and } p \neq q, \\
H\left(t-\left|\frac{a}{p}-\frac{a}{q}\right|\right) \text { if } p \text { and } q \text { are even or } p q=\infty, \\
H(t-3) \text { otherwise. }
\end{array}\right.
$$

It easy to show that $\left(M, F^{a}, T_{M}, 4\right)$ is a $b$-Menger space with $T_{M}$ is continuous. In the sequel, we take $a=1$. Consider the sequence $x_{n}=2 n, n \in \mathbb{N}$. Then $F_{2 n \infty}(t)=H\left(t-\frac{1}{2 n}\right)$. Therefore $x_{n} \rightarrow \infty$, but $F_{2 n 1}(t)=H(t-3) \neq H(t-1)=F_{1 \infty}(t)$. Hence $F$ is not continuous at $\infty$.

Let $f$ be a self map on $M$. Power of $f$ at $p \in M$ are defined by $f^{0} p=p$ and $f^{n+1} p=f\left(f^{n} p\right), n \geq 0$. We will use the notation $p_{n}=f^{n} p$, in particular $p_{0}=p, p_{1}=f p$.

Let $T$ be a t-norm, define the operator $T^{n}$ by

$T^{0}\left(x_{1}\right)=x_{1}$ and $T^{n}\left(x_{1}, x_{2}, \ldots, x_{n+1}\right)=T\left(T^{n-1}\left(x_{1}, x_{2}, \ldots, x_{n}\right), x_{n+1}\right)$ for all $n \geq 1$ and $x_{i} \in[0,1]$ for $i=1,2, \ldots, n+1$. 


\section{Main result}

Definition 3.1. [9] A function $\psi:[0,1] \rightarrow[0,1]$ is called a comparison function if it satisfies

(1) $\psi$ is nondecreasing and left continuous.

(2) $\psi(t)>t$ for all $t \in(0,1)$.

Lemma 3.1. [9] Let $\psi$ be a comparison function, then

(1) $\psi(1)=1$.

(2) $\lim _{n \rightarrow \infty} \psi^{n}(t)=1$ for all $t \in(0,1)$, where $\psi^{n}(t)$ denotes the composition of $\psi(t)$ with itself $n$ times.

Now, we can state and prove the main fixed point of this paper.

Theorem 3.1. Let $(M, F, T, s)$ be a G-complete $b$-Menger space under a continuous $t$-norm $T$ such that $F_{p q}(t)>0$ for each $p, q \in M$ and $t>0$. Let $A$ and $B$ be nonempty closed subsets of $M$ and let $f: A \cup B \rightarrow A \cup B$ be a mapping and satisfies

(1) $f(A) \subset B$ and $f(B) \subset A$.

(2) There exists a comparison function $\psi:[0,1] \rightarrow[0,1]$ such that

$$
F_{f p f q}(t) \geq \psi\left(F_{p q}(t)\right) \text {, }
$$

$\forall p \in A, \forall q \in B$ and $t>0$.

Then $f$ has a unique fixed point in $A \cap B$.

Proof. Let $p \in A \cup B$ and $t>0$, we have

$$
\begin{aligned}
F_{p_{n} p_{n+1}}(t) & =F_{f p_{n-1} f p_{n}}(t) \\
& \geq \psi\left(F_{p_{n-1} p_{n}}(t)\right) \\
& \vdots \\
& \geq \psi^{n}\left(F_{p_{0} p_{1}}(t)\right) .
\end{aligned}
$$

Thus, for any $r>0$, we have

$$
\begin{aligned}
F_{p_{n} p_{n+r}}(t) & \geq T\left(F_{p_{n} p_{n+1}}\left(\frac{t}{2 s}\right), F_{p_{n+1} p_{n+r}}\left(\frac{t}{2 s}\right)\right) \\
& \geq T\left(\psi^{n}\left(F_{p_{0} p_{1}}\left(\frac{t}{2 s}\right)\right), T\left(F_{p_{n+1} p_{n+2}}\left(\frac{t}{(2 s)^{2}}\right), F_{p_{n+2} p_{n+r}}\left(\frac{t}{(2 s)^{2}}\right)\right)\right. \\
& \geq T\left(T\left(\psi^{n}\left(F_{p_{0} p_{1}}\left(\frac{t}{2 s}\right)\right), \psi^{n+1}\left(F_{p_{0} p_{1}}\left(\frac{t}{(2 s)^{2}}\right)\right)\right), F_{p_{n+2} p_{n+r}}\left(\frac{t}{(2 s)^{2}}\right)\right) \\
& \vdots \\
& \geq T^{r-1}\left(\psi^{n}\left(F_{p_{0} p_{1}}\left(\frac{t}{2 s}\right)\right), \psi^{n+1}\left(F_{p_{0} p_{1}}\left(\frac{t}{(2 s)^{2}}\right)\right), \psi^{n+2}\left(F_{p_{0} p_{1}}\left(\frac{t}{(2 s)^{3}}\right)\right), \ldots, \psi^{n+r-1}\left(F_{p_{0} p_{1}}\left(\frac{t}{(2 s)^{r}}\right)\right) .\right.
\end{aligned}
$$

Since $F_{p_{0} p_{1}}(t)>0$ for all $t>0$, by Lemma 3.1, for every $i \in\{0,1,2, \ldots, r-1\}$, we obtain that

$$
\lim _{n \rightarrow \infty} \psi^{n+i}(t)=1 \text {. }
$$

By continuity of $t$-norm $T$, we get

$$
\lim _{n \rightarrow \infty} F_{p_{n+r} p_{n}}(t)=1 \text {. }
$$

It shows that $\left\{p_{n}\right\}$ is a $G$-Cauchy sequence in the $G$-complete subset $A \cup B$. So there exists $q \in M$ such that

$$
\lim _{n \rightarrow \infty} p_{n}=q .
$$

The subsequence $\left\{p_{2 n}\right\}$ is in either the set $A$ or in the set $B$ and a subsequence $\left\{p_{2 n+1}\right\}$ is in other set, and $A$ and $B$ are closed sets then $q \in A \cap B$. For any $t>0$, we have

$$
F_{f q q}(t) \geq T\left(F_{f q f p_{n}}\left(\frac{t}{2 s}\right), F_{f p_{n} q}\left(\frac{t}{2 s}\right)\right)
$$




$$
\geq T\left(\psi\left(F_{q p_{n}}\left(\frac{t}{2 s}\right)\right), F_{p_{n+1} q}\left(\frac{t}{2 s}\right)\right) \rightarrow T(1,1)=1 \text { as } n \rightarrow \infty
$$

Then

$$
f q=q \text {. }
$$

To show uniqueness, we assume that $u \in A \cap B$ is another fixed point of $f$ with $u \neq q$, we have

$$
\begin{aligned}
F_{q u}(t) & =F_{f q f u}(t) \\
& \geq \psi\left(F_{q u}(t)\right) \\
& >F_{q u}(t),
\end{aligned}
$$

which is a contradiction. Thus $q$ is the unique fixed point for $f$.

The following example support our result.

Example 3.1. Let $M$ be the subset of $\mathbb{R}^{2}$ defined by

$$
M=\{O, P, Q, R, S\}
$$

where $O=(0,0), P=(0,1), Q=(1,1), R=(0,2), S=(3,3)$.

Define

$$
F_{p q}(t)=\exp \left(-\frac{d(p, q)}{t}\right)
$$

where

$$
d(p, q)=\left(p_{1}-q_{1}\right)^{2}+\left(p_{2}-q_{2}\right)^{2}
$$

for all $p=\left(p_{1}, p_{2}\right) \in \mathbb{R}^{2}$ and $q=\left(q_{1}, q_{2}\right) \in \mathbb{R}^{2}$.

We have

$$
d(p, q) \leq 2(d(p, r)+d(r, q)) \quad \text { for all } p, q, r \in \mathbb{R}^{2}
$$

Then

$$
d(p, q) \leq 2\left(\frac{t_{1}+t_{2}}{t_{1}} d(p, r)+\frac{t_{1}+t_{2}}{t_{2}} d(r, q)\right) \quad \text { for all } p, q, r \in \mathbb{R}^{2} \text { and } t_{1}>0, t_{2}>0
$$

Then

So

$$
\frac{-d(p, q)}{t_{1}+t_{2}} \geq \frac{-2}{t_{1}} d(p, r)-\frac{2}{t_{2}} d(r, q) \quad \text { for all } p, q, r \in \mathbb{R}^{2} \text { and } t_{1}>0, t_{2}>0
$$

$$
\exp \left(\frac{-d(p, q)}{2\left(t_{1}+t_{2}\right)}\right) \geq \exp \left(\frac{-d(p, r)}{t_{1}}\right) \cdot \exp \left(\frac{-d(r, q)}{t_{2}}\right) \quad \text { for all } p, q, r \in \mathbb{R}^{2} \text { and } t_{1}>0, t_{2}>0
$$

So

$$
F_{p q}\left(2\left(t_{1}+t_{2}\right)\right) \geq T_{p}\left(F_{p r}\left(t_{1}\right), F_{r q}\left(t_{2}\right)\right) \quad \text { for all } p, q, r \in \mathbb{R}^{2} \text { and } t_{1}>0, t_{2}>0
$$

where $T_{p}$ is the $t$-norm product. Therefore, $\left(M, F, T_{p}, 2\right)$ is a $b$-Menger space.

Let $f: M \rightarrow M$ be given by

$$
f(O)=f(P)=f(Q)=f(R)=P, f(S)=O .
$$

Set $A=\{O, P, Q, R\}$ and $B=\{P, R, S\} . f(A) \subset B$ and $f(B) \subset A$.

In other hand, it can easily be verified that

$$
F_{f p f q}(t) \geq \psi\left(F_{p q}(t)\right) \quad \text { for every } p \in A \text { and } q \in B \text { and } t>0
$$

where $\psi(t)=\sqrt{t}$. In addition, $\left(M, F, T_{p}, 2\right)$ is a G-complete $b$-Menger space and $F_{p q}(t)>0$ for all $p, q \in M$ and $t>0$, then all the conditions of Theorem 3.1 are satisfied and then $f$ has a unique fixed point, that is $P \in A \cap B$. 


\section{References}

[1] Banach S., Sur les opérations dans les ensembles abstraits et leur applications aux équations intégrales. Fundam. Maths. 3, 133-181(1922)

[2] Cirić L., Solving the Banach fixed point principle for nonlinear contractions in probabilistic metric spaces, Nonlinear Analysis 72 (2010) 2009-2018.

[3] Kirk W. A., Srinivasan P. S., Veeramani, Fixed points for mappings satisfying cyclical contractive conditions. Fixed Point Theory, volume 4, No. 1, 2003, 79-89.

[4] Mbarki A., Oubrahim R., Probabilistic $b$-metric spaces and nonlinear contractions. Fixed Point Theory and Applications (2017) 2017:29

[5] Mbarki A., Oubrahim R., Cyclical contractive conditions in probabilistic metric spaces. Advances in Science, Technology and Engineering System Journal. Vol. 2, No. 5, 100-103 (2017).

[6] Menger K., Statistical metrics, Proc. Natl. Acad. Sci. 28 (1942), 535-537.

[7] Schweizer B. and Sklar A., Probabilistic Metric Spaces, North-Holland Series in Probability and Applied Mathimatics, 5, (1983).

[8] Sehgal V. M., and Bharucha-Reid A. T., Fixed points of contractions mappings on probabilistic metric spaces , Math. Systemes Theory, 6 (1972), 97-102.

[9] Shen Y.H., Qiu D., Chen W., Fixed point for cyclic $\varphi$-contractions in fuzzy metric spaces. Iranian Journal of Fuzzy Systems Vol. 10, No. 4,(2013) pp 125-133. 